ORIGINAL ARTICLE

\title{
Radiation exposure to personnel performing endoscopic retrograde cholangiopancreatography
}

\author{
L S Naidu, S Singhal, D E Preece, A Vohrah, D E Loft
}

Postgrad Med J 2005;81:660-662. doi: 10.1136/pgmj.2004.031526

See end of article for authors' affiliations

......................

Correspondence to: Dr L S Naidu, Department of Radiology, University Hospitals of Leicester NHS Trust, Leicester LE1 5WW, UK; srinaidu@hotmail.com

Submitted

12 December 2004

Accepted

17 February 2005
Background: Endoscopic retrograde cholangiopancreatography (ERCP) relies on the use of ionising radiation but risks to operator and patient associated with radiation exposure are unclear. The aim of this prospective study was to estimate the radiation dose received by personnel performing fluoroscopic endoscopic procedures, mainly ERCP.

Methods: Consecutive procedures over a two month period were included. The use of thermoluminescent dosimeters to measure radiation exposure to the abdomen, thyroid gland, and hands of the operator permitted an estimation of the annual whole body effective dose equivalent.

Results: During the study period 66 procedures (61 ERCP) were performed and the estimated annual whole body effective dose equivalent received by consultant operators ranged between 3.35 and $5.87 \mathrm{mSv}$. These values are similar to those received by patients undergoing barium studies and equate to an estimated additional lifetime fatal cancer risk between 1 in 7000 and 1 in 3500 . While within legal safety limits for radiation exposure to personnel, these doses are higher than values deemed acceptable for the general public.

Conclusions: It is suggested that personnel as well as patients may be exposed to significant values of radiation during ERCP. The study emphasises the need to carefully assess the indication for, and to use measures that minimise radiation exposure during any fluoroscopic procedure.
E ndoscopic retrograde cholangiopancreatography (ERCP) is an invasive technique that has been used for over 30 years in the diagnosis and management of pancreaticobiliary disorders. With the advent in recent years of noninvasive diagnostic techniques such as magnetic resonance cholangiopancreatography and endoscopic ultrasonography, there has been a decreasing requirement for diagnostic but an increasing requirement for therapeutic ERCP. There is a significant complication rate associated with this procedure; thus, a recent multicentre study prospectively evaluated 2444 ERCP procedures and reported an overall complication rate of $5 \% .{ }^{1}$ The main complications, in order of frequency, were pancreatitis $(1.8 \%)$, haemorrhage $(1.1 \%)$, cholangitis $(0.6 \%)$, perforation $(0.6 \%)$, and death $(0.2 \%)$.

ERCP relies heavily on the use of radiographs, but the risks associated with radiation exposure (both to patients undergoing and to personnel undertaking the procedure) have not been extensively studied. Of particular concern is the potential additional lifetime risk of fatal cancer from radiation exposure, but the short follow up period of studies such as that described above precludes an assessment of this risk. When ionising radiation is absorbed by living tissues, chemical changes occur immediately, molecular damage occurs within minutes, but biological damage (in the form of inhibition of cell division, cellular transformation to a malignant state, or cell death) becomes evident only after a much longer period (up to many decades). ${ }^{2}$ The pioneering UK radiologist, Dr Hall-Edwards, calibrated exposure from early $x$ ray tubes in the late 1800 s by placing his right arm under the emitting source; he subsequently developed severe radiation dermatitis and a squamous cell carcinoma on the dorsum of his right hand that required amputation.

The estimated risk associated with exposure to ionising radiation is based on the effective dose (ED) received, for which the unit of measurement is the Sievert (Sv). For any radiological procedure the ionising dose absorbed by a particular tissue or organ in the irradiated person is first calculated (in milligrays). A radiation weighting factor that takes into account the type of radiation is then used to calculate the "equivalent dose" for that tissue or organ. Lastly, a tissue weighting factor is used to take account of the fact that the risk of biological damage to the whole body varies according to the tissue or organ irradiated. The ED is then calculated as the sum of the weighted equivalent doses for all the tissues or organs that have been exposed and is expressed as the whole body ED equivalent. Thus, if a person is uniformly irradiated by $x$ rays then the whole body absorbed dose, equivalent dose, and ED will be the same.

The lifetime risk of fatal cancer after exposure to ionising radiation has been estimated as $6 \%$ per Sv. ${ }^{3}$ Typical whole body ED equivalents received by patients undergoing radiographic procedures have been calculated, ${ }^{4}$ including chest radiograph $(0.02 \mathrm{mSv})$, barium enema $(7 \mathrm{mSv})$, and abdominal computed tomography $(10 \mathrm{mSv})$; these values correlate with estimated lifetime fatal cancer risks of 1 in 670000,1 in 4800 , and 1 in 3300 respectively. These risks are additional to the 1 in 5 lifetime risk of fatal cancer for the general population. It should be noted that each person in the UK annually receives an estimated radiation dose of $2.6 \mathrm{mSv}$, of which $85 \%(2.2 \mathrm{mSv})$ comes from natural background radiation (mainly radon gas). Ionising medical radiation accounts for most of the radiation from artificial sources $(0.37 \mathrm{mSv}$ of $0.4 \mathrm{mSv})$. $^{5}$

We performed a prospective study in a teaching hospital that performs more than 400 ERCPs annually, in an attempt to estimate the radiation dose (annual whole body ED equivalent) received by personnel undertaking fluoroscopic endoscopic procedures. We compared the data obtained with legal safety limits for maximum exposure.

Abbreviations: $E R C P$, endoscopic retrograde cholangiopancreatography; $E D$, effective dose 
Table 1 lonising Radiation Regulations (1999) annual $\mathrm{ED}$ equivalent limits ${ }^{6}$

\begin{tabular}{llll}
\hline & \multicolumn{3}{l}{ Dose limits $(\mathbf{m S v})$} \\
\cline { 2 - 4 } & $\begin{array}{l}\text { Staff } \\
>\mathbf{1 8} \text { years }\end{array}$ & $\begin{array}{l}\text { Trainees aged } \\
\leqslant \mathbf{1 8} \text { years }\end{array}$ & Public visitors \\
\hline $\begin{array}{l}\text { Whole body } \\
\text { Eyes }\end{array}$ & 20 & 6 & 1 \\
$\begin{array}{l}\text { Extremities and other } \\
\text { organs }\end{array}$ & 150 & 50 & 15 \\
& & 150 & 50 \\
ED, effective dose; $\mathrm{mSv}$, milliSievert. & & \\
\hline
\end{tabular}

\section{METHODS}

Local research ethics committee approval was obtained. Consecutive endoscopic procedures requiring fluoroscopy (usually ERCP) over a two month period starting November 2000 were included in the study. During the study period one of three consultants (each with similar endoscopic experience) and/or one of two specialist registrars with access to a total of three fluoroscopy sessions per week either performed or assisted at each procedure.

The three consultant operators had one session per week with three to four ERCP/session. There was no deviation from standard unit practice either with regard to patient consent or in terms of the procedures performed.

The procedures were performed in the radiology department using (for fluoroscopy purposes) a CGR Prestilix 1600exponent 100 IST (Campagnia Generale Di Radiologia, Monza, Italy). An "over-couch" Comet $150 \mathrm{kV} x$ ray emitter tube (Comet AG, Switzerland) was used for image intensification and for the purposes of taking plain films. The automatic pre-set values for fluoroscopy voltage and current were $80 \mathrm{kV}$ and $400 \mathrm{~mA}$ respectively with an exposure time in the range $0.04-0.1$ seconds. These values are adjusted automatically (for example, according to patient build) to maintain a constant radiation dose entering the image intensifier.

The operator wore a $0.25 \mathrm{~mm}$ lead equivalent thick apron and three thermoluminescent dosimeters (Lithium Fluoride TLD 100, Regional Radiation Physics and Protection Service (RRPPS), Birmingham, England) at the following sites: beneath lead apron, left collar, and left middle finger, to measure radiation exposure respectively to the abdomen, thyroid gland, and extremities.

For each examination the type of procedure, total fluoroscopy time, total number of radiographs taken, and fluoroscopy tube voltage and current were recorded. Previous studies have estimated radiation dose emission from the $x$ ray source directly using a DAP meter (see above) but, as in many units undertaking ERCP the equipment we used was not fitted with a DAP meter at the time of this study. We therefore estimated the emitted radiation dose indirectly from the fluoroscopic exposure time, the fluoroscopy tube current, and the number of radiogarphs taken. The thermoluminescent dosimeters were analysed by the RRPPS and the ED for each operator during the two month study period was

Table 2 Mean (SD) number of radiographs taken by each operator

\begin{tabular}{lll}
\hline & Diagnostic procedure & Therapeutic procedure \\
\hline C1 & $3.7(1.64)$ & $4.6(1.74)$ \\
C2 & $5.2(1.3)$ & $1.8(1.33)$ \\
C3 & $4.1(2.71)$ & $2.7(2.15)$ \\
R1 & $3.8(1.6)$ & $4.8(1.98)$ \\
R2 & $4.8(0.95)$ & $1.6(1.01)$ \\
\hline \multicolumn{2}{l}{ C, consultant; R, specialist registrar. } \\
\hline
\end{tabular}

Table 3 Extrapolated annual ED ( $\mathrm{mSv}$ ) to the abdomen, thyroid gland, and extremities for each operator

\begin{tabular}{llll}
\hline & Abdomen & Thyroid gland & Extremities \\
\hline C1 & 3.6 & 82.2 & 14.4 \\
C2 & 3.0 & 25.8 & 9.0 \\
C3 & 1.8 & 56.4 & 8.4 \\
R1 & 0 & 19.8 & 4.2 \\
R2 & 0 & 16.2 & 3.6 \\
\hline \multirow{2}{*}{ ED, effective dose; $m$ mSv, milliSievert; C, consultant; $R$, specialist registrar. }
\end{tabular}

Table 4 Annual whole body ED equivalent for each operator

\begin{tabular}{ll}
\hline \multicolumn{2}{c}{ ED (mSv) } \\
\hline C1 & 5.87 \\
C2 & 3.73 \\
C3 & 3.35 \\
R1 & 0.54 \\
R2 & 0.43 \\
\hline ED, effective dose; mSv, milliSievert; C, consultant; R, \\
specialist registrar.
\end{tabular}

calculated. These figures were extrapolated to estimate the annual whole body ED equivalent received and the data were compared with legal safety limits for maximum annual exposure as defined by the Royal College of Radiologists and the National Radiological Protection Board (Ionising Radiation Regulations, 1999; table 1). ${ }^{6}$

\section{RESULTS}

During the study period 66 fluoroscopic endoscopic procedures were performed on 66 patients, of whom 61 underwent ERCP (23 diagnostic, 38 therapeutic), four underwent oesophageal procedures, and one underwent a pancreatic biopsy.

The mean (SD) fluoroscopic time was 3.55 (1.81) minutes for diagnostic and 5.67 (4.09) minutes for therapeutic ERCP. Mean fluoroscopic times for therapeutic ERCP with and without papillotomy were 5.97 (3.75) and 4.28 (3.41) minutes respectively.

Table 2 shows the mean number of radiographs taken by each operator.

Table 3 shows the estimated radiation exposure (annual ED extrapolated from two month figures as calculated by the RRPPS) to the abdomen, thyroid gland, and extremities for each operator.

Webster's formula ${ }^{7}$ was then used to estimate the annual whole body ED equivalent (He):

$$
\mathrm{He}=\frac{(1.51 \times \mathrm{H} 1)+(0.04 \times \mathrm{H} 2)}{1.48}
$$

where $\mathrm{Hl}$ is the annual exposure to the abdomen and $\mathrm{H} 2$ is the annual dose to the thyroid gland. Table 4 shows the annual whole body ED equivalent for each operator.

\section{DISCUSSION}

The findings from this prospective study suggest that the operator at ERCP is exposed to significant doses of radiation. The estimated annual whole body ED equivalent values calculated for the three consultant operators (3.35-5.87 mSv) are similar to those received by patients undergoing fluoroscopic procedures including barium studies (estimated additional lifetime fatal cancer risk between 1 in 7000 and 1 in 3500). While these values are within the legal safety limits for whole body radiation exposure to personnel, they are higher than the levels deemed acceptable for "public 
visitors" (table 1). The dose limits shown are legal limits and staff who are likely to exceed $30 \%$ of any of the annual dose limits (that is, $6 \mathrm{mSv}$ for whole body ED equivalent) should be designated as "classified". All classified persons are required to wear personal dosimeters while undertaking fluoroscopic procedures and their dose and medical records should be kept centrally by the Health and Safety Executive for 50 years after the last entry. ${ }^{2}$

Radiation exposure to the thyroid gland seems to be of particular concern (25.8-85.2 mSv estimated annual ED received by the three consultant operators, table 3 ), although the values recorded are within the legal safety limits for radiation exposure to the eyes, extremities, and other organs (table 1). Furthermore, the contribution to the whole body ED equivalent is adjusted for each tissue or organ according to Webster's formula (see results). This formula uses a tissue weighting factor (see above) to take account of non-uniform irradiation and in particular reflects the fact that radiation exposure to particular tissues (for example, intra-abdominal organs) contributes disproportionately heavily to the total estimated additional lifetime fatal cancer risk. Thus, radiation exposure to the thyroid gland is "scaled down" by a factor of 25 while that to the abdomen is "scaled up" by a factor of 1.51 .

Few data exist regarding the radiation related risks to personnel performing ERCP. It is known that the patient is a source of scattered radiation and that the radiation dose received by the operator is proportional to that received by the patient. Studies confirm that the radiation exposure to personnel undertaking ERCP is related to the duration of fluoroscopy, ${ }^{8} 9$ and that this exposure may be reduced by the use of protective lead shielding ${ }^{810}$ and by the use of higher voltage and lower current for fluoroscopy. ${ }^{9}$

Larkin et al did attempt to estimate the ED of ionising radiation received by patients (but not by personnel) undergoing ERCP. ${ }^{11}$ In that study the radiation dose emitted by the $x$ ray source was estimated from the dose area product (DAP, Gray per $\mathrm{cm}^{2}$ ), measured using a transmission ionising chamber attached to the collimator box of the $x$ ray fluoroscopy tube. The ED for each procedure was estimated from the DAP, the fluoroscopy tube voltage and current, and the number of radiographs taken. For 20 consecutive ERCPs the mean ED was $3.1 \mathrm{mSv}$ (estimated additional lifetime fatal cancer risk 1 in 6700) for diagnostic and $12.4 \mathrm{mSv}$ (estimated additional fatal lifetime cancer risk 1 in 1700) for therapeutic procedures. Importantly, the radiation dose received showed a linear correlation with fluoroscopy (screening) time but not with the number of radiographs taken.

The International Commission on Radiological Protection emphasises the need for carefully assessing the indication for any fluoroscopic procedure ("justification") to ensure that the benefit of the procedure is perceived to be greater than the risk (to staff, visitors, or patients) from radiation exposure. This study suggests that personnel as well as patients can be exposed to significant levels of radiation, and personnel are at particular risk during procedures such as ERCP, at which the operator remains in close contact with the patient throughout the period of screening. For both operator and patient a DAP meter is the recommended modality for measuring radiation exposure during fluoroscopic screening.

The United States National Council on Radiation Protection and Measurements highlighted mechanisms by which a strategy for minimising radiation to personnel ("optimisation") could be implemented. ${ }^{12}$ The design of the fluoroscopic system is particularly important. Thus, an "under-couch" $x$ ray emitter tube (as compared with the "over-couch" tube used in our unit during this study) more effectively ensures that scattering of the emitted radiation occurs when the primary beam makes contact with the patient and hence safer. Protective lead shielding $(0.5 \mathrm{~mm}$ lead equivalent thick) between the operator and $x$ ray emitter should be available and has been shown to reduce the amount of scattered radiation that the operator is exposed to. ${ }^{58}$ Next, with increasing distance (r) from the $x$ ray source the radiation dose is reduced according to the inverse square law $\left(1 / \mathrm{r}^{2}\right)$; thus, doubling the distance from the source reduces the radiation dose received by a factor of 4 . The operator and radiographer should, therefore, stand back from the patient during exposure and also, where possible, screen intermittently and make use of coning techniques. Protective clothing protects against radiation scattered by the patient and the standard $0.25 \mathrm{~mm}$ lead equivalent thick aprons commonly used transmit only $10 \%$ of radiation scattered at $90^{\circ}$. Lastly, this study suggests that radiation exposure to the thyroid gland may be particularly significant and the use of a thyroid protection shield (not currently used by all operators undertaking ERCP) is recommended.

Subsequent to the data obtained from this study, practice has been modified in our department. The radiation risks are emphasised at the outset to all staff (medical and nursing) undertaking ERCP procedures, and all involved personnel wear a thyroid protector. Some operators (particularly the nurse assistant monitoring the airway at the head of the patient) additionally wear lead goggles. The endoscopy nursing staff are rotated between ERCP and other endoscopic procedures on a regular basis, with strict dosimetric monitoring. Within each ERCP session, the nurse assistants rotate so that the same nurse is not situated at the head of the patient or nearer the primary beam throughout the session.

\section{CONCLUSION}

We suggest that personnel as well as patients may be exposed to significant levels of radiation during ERCP. We emphasise the need to carefully assess the indication for, and to use measures that minimise radiation exposure during any fluoroscopic procedure.

\section{Authors' affiliations}

L S Naidu, S Singhal, D E Loft, Department of Gastroenterology, University Hospitals Coventry and Warwickshire NHS Trust, UK

D E Preece, Department of Clinical Physics and Bioengineering,

University Hospitals Coventry and Warwickshire NHS Trust

A Vohrah, Department of Radiology, University Hospitals Coventry and Warwickshire NHS Trust

Funding: none.

Conflicts of interest: none.

\section{REFERENCES}

1 Masci E, Toti G, Mariani A, et al. Complications of diagnostic and therapeutic ERCP: a prospective multicenter study. Am J Gastroenterol 2001;96:417-23.

2 Farr RF, Allisy-Roberts PJ. Physics for medical imaging. London: W B Saunders, 1998:148-81.

3 NRPB. Estimates of late radiation risks to the UK population. Irradiation in utero. London: NRPB, 1993:105-25.

4 International Commission on Radiological Protection. 1990 Recommendations of the International Commission on Radiological Protection. ICRP Publication 60. Oxford: Pergamon Press, 1991:1-3.

5 Hughes JS. lonising radiation exposure of the UK population. London: NRPB, 1999.

6 RCR/NRPB. The ionising radiation regulations 1999. London: The Stationery Office, 1999.

7 Webster EW. EDE for exposure with protective aprons. Health Phys 1989;56:568-9.

8 Chen MY, Van Swearingen FL, Mitchell R, et al. Radiation exposure during ERCP: effect of a protective shield. Gastrointest Endosc 1996;43:1-5.

9 Heyd RL, Kopecky KK, Sherman S, et al. Radiation exposure to patients and personnel during interventional ERCP at a teaching institution. Gastrointest Endosc 1996;44:287-92.

10 Cohen RV, Aldred MA, Paes WS, et al. How safe is ERCP to the endoscopist? Surg Endosc 1997;11:615-17.

11 Larkin CJ, Workman A, Wright RER, et al. Radiation doses to patients during ERCP. Gastrointest Endosc 2001;53:161-4.

12 NCRP. Implementation of the principle of as low as reasonably achievable (ALARA) for medical and dental personnel. Bethseda: National Council on Radiation Protection and Measurements, 1990. 